\title{
Impact of Packet Size on the Performance Evaluation of Routing Protocols in Cognitive Radio Networks
}

\author{
Sopan Talekar \\ Research Scholar \\ P D A College of Engineering \\ Kalaburagi, Karnataka
}

\author{
Sujatha Terdal \\ Professor \\ P D A College of Engineering \\ Kalaburagi, Karnataka
}

\begin{abstract}
Cognitive Radio Network (CRN) is a next generation of wireless communication. Cognitive Radio (CR) is able to detect the unoccupied spectrum and allow to secondary users (SU) also known as cognitive users (CU) to use it. In order to improve the performance of cognitive radio network, many research has been done in routing protocols.

In this paper, the scenario of impact of packet size on two routing protocols are evaluated: i) Routing protocol with Machine Learning (ML) and ii) Routing protocol without Machine Learning (ML). While transmitting packet from sender to receiver, packet size plays an important role in the performance of network.

The above two scenario have been implemented in NS2 network simulator. This paper analyses the impact of various packet size on routing protocol in cognitive radio network. Routing protocol with and without ML approach with various packet size is compared and evaluated performance parameters like delay, dropping ratio and packet delivery ratio.
\end{abstract}

\section{General Terms}

Cognitive Radio Network, Machine Learning.

\section{Keywords}

CRN routing protocol, Packet size, Routing metrics, Network Simulator (NS2).

\section{INTRODUCTION}

Joseph Mitola III has proposed the concept of Cognitive Radio (CR). According to the proposal, CR was an innovative approach in wireless communication [1].

$\mathrm{CR}$ is able to detect unused or unoccupied spectrum and assign to SU to use it. Hence it solves the problem of limited spectrum as well as underutilized spectrum.

The Federal Communications Commission (FCC) provide the guideline about use of radio spectrum [2]. According to it, spectrum is categorize into licensed and unlicensed spectrum. Licensed spectrums are assigned to Primary Users (PU) for specific service and are underutilized whereas unlicensed spectrums are assigned to SUs of various technologies like Bluetooth, Wi-Fi, Cordless Phones etc. which are always over crowded. Hence unlicensed spectrum is not enough to provide the better service to the SUs. Therefore, SUs are allowed to utilize the licensed spectrums when it is not occupied by PUs based on dynamic spectrum access (DSA) techniques [3].

Most of the research work has been done in the PHY \& MAC layer of CRN, but very limited work has been done so for in the network layer (Routing layer) of Cognitive Radio Network
The existing routing techniques and protocol of MANET are not convenient to CRN. MANET routing protocol mostly use hop count and delay as routing metric but it is not sufficient in CRN to improve the performance of routing. Therefore, different routing metrics such as power consumption, route stability, PU/SU interference, spectrum awareness etc. are taken into consideration to design CRN routing protocol [4] [5]. In order to improve the performance of routing, it is necessary to select appropriate routing metric, channel selection strategy as well as packet size.

The rest of the paper is organized as follow. Section 2 includes related work of routing protocols in CRN. Section 3 describes design of routing protocol. Section 4 discusses the simulation and performance metrics. Performance evaluation of CRN routing protocol is discussed in section 5. Finally, the work is concluded in section 6 .

\section{RELATED WORK}

Several routing protocols in Cognitive radio network have been proposed. Performance of most of the routing protocols have been evaluated by considering number of nodes, number of channels, number of PUs/SUs etc.

Most of the work is carried out for fixed packet size and hence performance of routing protocol is not evaluated with different packet size. Variable packet size is also impacts on the performance of routing. Small size packet induce more overhead while large size packet induce more delay in packet transmission but helps to reduce the overhead. Therefore, it is very important to select appropriate size of packets for data transmission. In the work [6] [7] [8] [9], variable packet size is not consider to evaluate the performance of routing protocol.

Shelly Salim et.al proposed different on demand routing protocols for cognitive radio ad hoc networks which are classified based on AODV, DSR, and hybrid on-demand protocols. AODV is probably most suitable for cognitive wireless networks compared to DSR. Because DSR route discovery may lead to unpredictable packet length which is not suitable for intermittent connectivity environment of cognitive radio networks [6]. Ruchi Rai et.al presented work to address the problem of efficient route selection between source and destination. In this work, two routing protocols such as AODV \& WCETT in Cognitive Radio Ad-Hoc Networks (CRAHN) are compared. AODV uses hop count as metric whereas WCETT uses ETT (expected transmission time on link) metric. It is found that, due to the better route selection strategy, WCETT performs better than AODV. In this work, various routing models such as multi-radio, multichannel, equal number of radios $\&$ channels and single radio, multi-channel are not taken into consideration for the performance analysis [7]. Sajjad Rahmani et.al studied effect 
of MAC and routing protocol on TCP and improve the performance of transport layer protocol TCP with some changes in MAC protocols in cognitive radio network. Simulation results for AODV, DSDV and DSR routing algorithms for different MAC are discussed [8].

Yuting Wang et.al proposed a joint channel selection and routing protocol (CSRP) to ensure stability and reduce route latency between CUs. Historical information of channel availability and the channel switching delay are used for channel selection. Routing is performed based on shortest route which have high data delivery probabilities and low delays [9]. M.C.Oto and O.B.Akan determined the energy efficient optimal packet size based on the PU behavior and channel BER parameters for cognitive radio sensor networks (CRSN) [10]. Mohammed Al-Medhwahi, et.al [11], examined the performance of small, medium and large packet size in terms of delay and throughput in cognitive radio sensor network. The results show that the medium size packet performs better than the small and large size packet size. In this work, variable packet size is considered within an efficient MAC protocol such as Pliable Cognitive MAC (PCMAC) which is proposed and modelled in [12].

The work of variable packet size impact on routing protocol of cognitive radio network is not found. Therefore, this paper focuses more on impact of variable packet size on the performance of CRN routing protocol. In this work, the scenario of impact of packet size on two routing protocols are evaluated: i) Routing protocol with Machine Learning (ML) and ii) Routing protocol without Machine Learning (ML).

In the first scenario, Routing protocol is designed using machine learning (ML) strategy called as Reinforcement Learning (RL). In this routing protocol, RL is used to select best channel with maximum reward for the packet transmission. In the second scenario, routing protocol randomly selects channel from the available list and perform routing. It does not select optimal channel because it works without using any channel selection strategy.

\section{DESIGN OF ROUTING PROTOCOL}

\subsection{Use of Machine Learning in CRN}

In the design of routing protocol, channel is selected for routing using Reinforcement Learning (RL) as a type of Machine Learning (ML). Figure 1 shows the Basic model of RL [13], in which agent performs the channel selection as an action based on the current state of the environment and return the reward to the action performed. Agent learns from the observation and takes the optimal decision for the next state. The performance analysis of routing protocol with and without RL is evaluated for the fixed size of packets i.e. 1000 bytes [14].

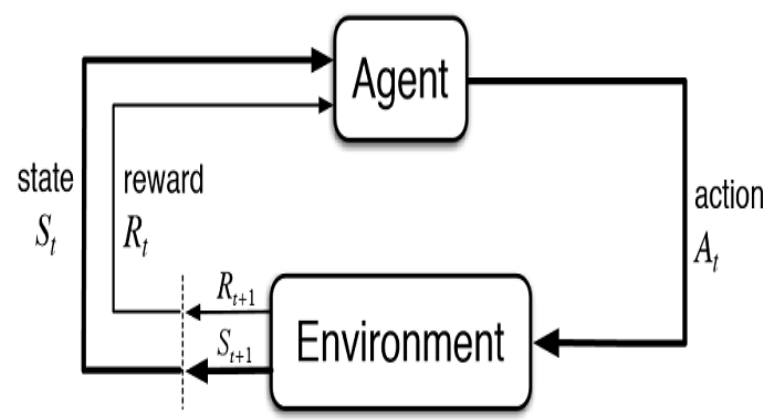

Figure 1: Basic RL Model
In the proposed work, performance of routing protocol is evaluated for variable packet size.

In the design of routing protocol, routing table plays vital role and keep the necessary information for routing. If SUs are within the communication range, they can directly communicate with each other without performing routing. In other case, routing plays an important role when SUs are not within the communication range and are unable communicate each other directly. Hence transmission of data between such SUs takes place through intermediate nodes.

Routing protocol in cognitive radio network determines a route to a destination only when a CU wants to send a packet to that destination. Routes are maintained until source finish its transmission. Routing protocol uses sequence numbers that determine the timeliness of each packet and ensures loop free routing.

\subsection{Route Discovery Process}

Each routing table entry contains the following information: Destination, next hop, number of hops, last hop count, destination sequence number, and active neighbors for this route and expiration time for this route table entry. Expiration time, also called lifetime, is reset each time the route has been used.

\section{Route Flags:}

If flag=0: Route Down, if flag=1: Route up and if flag=2: Route in repair.

Route request timeout (when I can send another request)

Route request count (Number of route requests)

The routing table of node store information about next hop to the destination and a sequence number. It is obtained when RREQ packet broadcast to its neighbors.

Intermediate node updates its routing table for a reverse path to the source when it receive a RREP packet. It is also update the forward path to the destination after receiving RREQ.

The fields of RREQ and RREP are shown in table 1 and table 2 respectively.

Various new fields are included in RREP control packet. X position and $\mathrm{Y}$ position describe the nodes position which is used to determine the distance from the primary user. Active user-list field keep the list of active users in the communication range and Active user count that counts the total number of active users in their communication range.

Timestamp field of both RREQ and RREP is used to compute route discovery latency.

Lifetime indicates that the entry of route will be deleted if it is not used within the particular lifetime.

Table 3 shows the fields of RERR. All nodes monitor their neighbors. It generates RERR (route error message) when a node is an unreachable in an active route and inform to all other nodes.

HELLO message is used for detecting and monitoring links to its neighbors.

\section{SIMULATION AND PERFORMANCE METRICS}

\subsection{NS2 Simulation Environment}

NS2 stands for Network Simulator with version 2. It is an open-source event-driven simulator. It is developed 
particularly for research in computer communication network. It consist of two languages C++ and OTCL. C++ is used at the backend of the simulation while OTCL is used to setup the simulation. Xgraph is used to display result and NAM is used to visualize the traffic flow.

The performance evaluation work is carried out using NS2 simulator with CRCN patch [15].

Table 1. RREQ Packet

\begin{tabular}{|c|c|c|c|c|c|c|c|}
\hline $\begin{array}{c}\text { Packet } \\
\text { Type }\end{array}$ & $\begin{array}{c}\text { Source IP } \\
\text { address }\end{array}$ & $\begin{array}{c}\text { Broadcast } \\
\text { ID }\end{array}$ & $\begin{array}{c}\text { Source } \\
\text { Sequence Number }\end{array}$ & $\begin{array}{c}\text { Destination IP } \\
\text { Address }\end{array}$ & $\begin{array}{c}\text { Destination } \\
\text { Sequence Number }\end{array}$ & $\begin{array}{c}\text { Hop } \\
\text { count }\end{array}$ & $\begin{array}{c}\text { Timestamp } \\
\end{array}$ \\
\hline
\end{tabular}

Table 2. RREP Packet

\begin{tabular}{|c|c|c|c|c|c|c|c|c|c|c|c|}
\hline $\begin{array}{l}\text { Packet } \\
\text { Type }\end{array}$ & $\begin{array}{c}\text { Source } \\
\text { IP } \\
\text { address }\end{array}$ & $\begin{array}{l}\text { Sequence } \\
\text { Number }\end{array}$ & $\begin{array}{l}\text { Destination } \\
\text { IP } \\
\text { Address }\end{array}$ & $\begin{array}{c}\text { Destination } \\
\text { Sequence } \\
\text { Number }\end{array}$ & $\begin{array}{c}\text { Hop } \\
\text { count }\end{array}$ & Lifetime & $\begin{array}{l}\text { Time } \\
\text { stamp }\end{array}$ & $\begin{array}{c}\mathrm{X} \\
\text { Pos. }\end{array}$ & $\begin{array}{c}\mathrm{Y} \\
\text { Pos. }\end{array}$ & $\begin{array}{c}\text { Active } \\
\text { User- } \\
\text { list }\end{array}$ & $\begin{array}{c}\text { Active } \\
\text { user } \\
\text { count }\end{array}$ \\
\hline
\end{tabular}

Table 3. RERR Packet

\begin{tabular}{|l|l|l|l|}
\hline Packet Type & Destination Count & $\begin{array}{l}\text { Unreachable Destination Address } \\
\text { List }\end{array}$ & $\begin{array}{l}\text { Unreachable Destination Sequence } \\
\text { Number List }\end{array}$ \\
\hline
\end{tabular}

\subsection{Simulation Parameters}

Simulation parameters for the work of routing protocols in Cognitive radio networks is shown in table 4.

Table 4. Simulation Parameters

\begin{tabular}{|c|c|}
\hline Parameters & Values \\
\hline Routing Protocols & $\begin{array}{c}\text { With_ML_CRP, } \\
\text { without_ML_CRP }\end{array}$ \\
\hline Simulation area & 600m x500m \\
\hline Number of nodes & 70 \\
\hline Number of PU & Random Way Point \\
\hline Mobility Model & Constant Bit Rate(CBR) \\
\hline Traffic model & Two Ray \\
\hline Pathloss model & Initial Energy:100 Joules \\
\hline Energy Model & $1000,1050,1100,1150,1200$ \\
\hline Packet Sizes in bytes & \\
\hline
\end{tabular}

\subsection{Performance Metrics}

\subsubsection{Delay}

It is defined as the time taken for a data packet to be transmitted from the source to destination. It includes delays as delay for route discovery, propagation time, data transfer time, intermediate queueing delay.

\subsubsection{Dropping Ratio}

It is the ratio of number of lost packets to the number of packets sent by the source.

\subsubsection{Packet Delivery Ratio}

It is the ratio of number of packets received by the destination to the number of packets sent by the source. It is measure in percentage.

\section{PERFORMANCE EVALUATION OF CRN ROUTING PROTOCOLS}

The simulation results of the ML-CRP and without_ML_CRP protocol in CRN under variable packet size are generated in the NS2 output trace files.

Results are analyzed using the NS2 Xgraph. The results are shown in the form of line graphs based on the performance metrics including delay, dropping ratio and packet deliver ratio.

Figure 2 shows that, there is a variation in delay performance parameter in ML_CRP as the packet size increases. But there is a not too much variation in delay in without_ML_CRP with change in packet size. In delay comparison, ML_CRP performs better than without_ML_CRP.

At the packet size 1050 bytes, ML_CRP protocol performs better compared to other packet size.

Figure 3 shows that, the performance parameter dropping ratio in ML_CRP is increases as the packet size increases. But there is a variation in dropping ratio in without_ML_CRP with change in packet size.

In dropping ratio comparison, ML_CRP performs better than without_ML_CRP. For lower packet size 1000 bytes, packet loss in ML_CRP is lowest in comparison with other packet size such as 1050, 1100, 1150 and 1200 bytes.

Figure 4 shows that, the performance parameter packet delivery ratio (PDR) in ML_CRP is decreases as the packet size increases. It is observed that, ML_CRP performs better packet delivery ratio for small size packet 1000 bytes. But there is a variation in packet delivery ratio (PDR) in without_ML_CRP with change in packet size.

In PDR comparison, ML_CRP performs better than without_ML_CRP. 


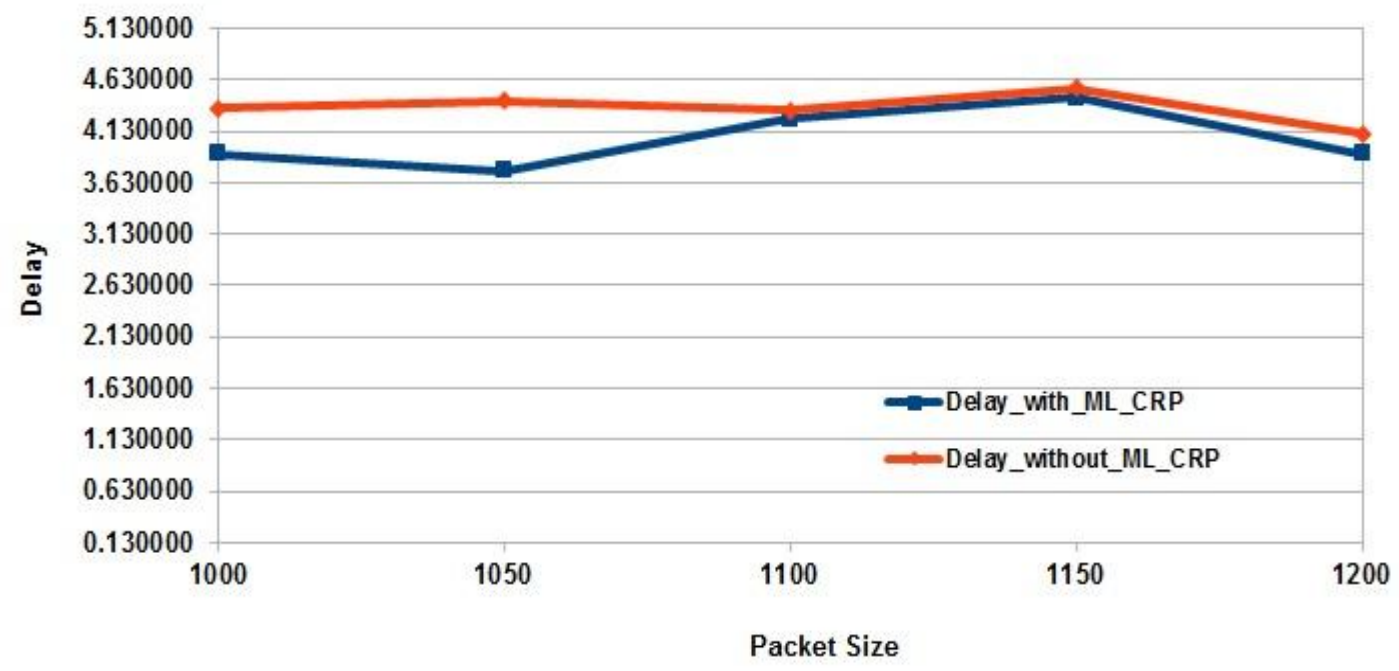

Figure 2. Packet size (bytes) vs Delay

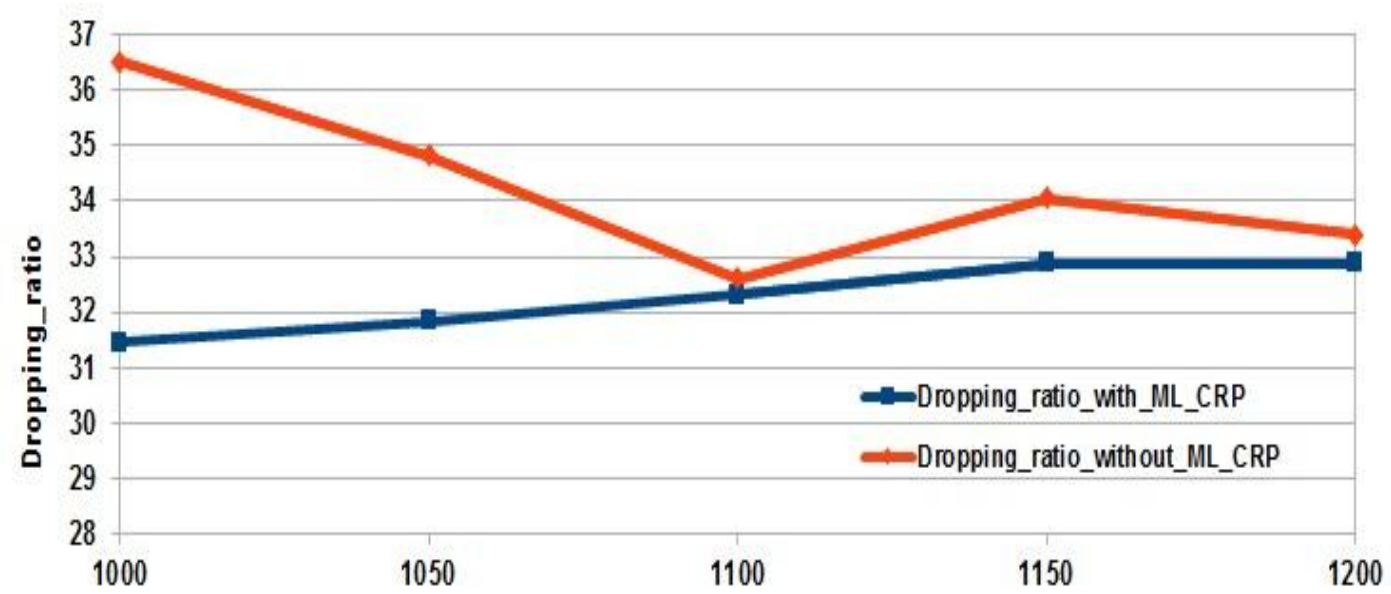

Packet Size

Figure 3. Packet size (bytes) vs Dropping ratio (\%)

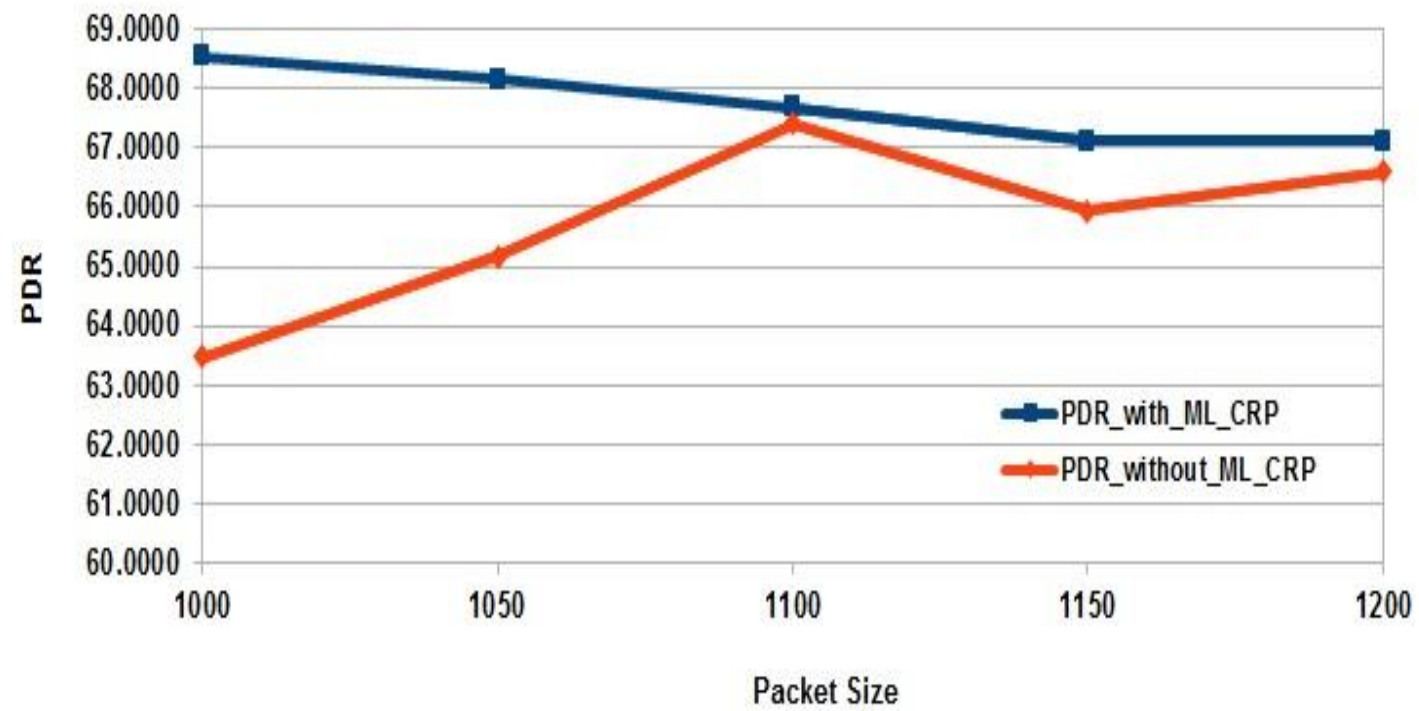

Figure 4. Packet size (bytes) vs PDR (\%) 


\section{CONCLUSION}

In this paper, Impact of packet size on routing protocol in cognitive radio network is analyzed. The performance of routing protocol is evaluated with variable packet size. From the results, it is observed that, packet size plays a vital role in the performance of network. In order to improve the performance of routing, it is significant to consider both channel selection strategy and appropriate packet size.

The simulation result shows that ML_CRP performs better than that of Without_ML_CRP routing protocol. It is observed that ML_CRP has less delay, better packet delivery ratio and least number of packet loss compared to Without_ML_CRP.

\section{REFERENCES}

[1] Mitola J, Maguire G.Q. Jr, "Cognitive radio: making software radios more personal", Personal Communication, IEEE, Vol 6.no 4, pp 13-18 Aug 1999.

[2] Federal Communications Commission, Spectrum policy Task force Report, ET Docket No.03-222, Notice of Proose Rule making \& order 2003.

[3] B. Wang, KJR Liu, "Advances in Cognitive Radio Networks: A Survey" IEEE Journal of Selected topics in signal processing, Vol.5.pp. 5-23, 2011.

[4] Moustafa Youssef, Mohamed Ibrahim, Mohamed Abdelatif, Lin Chen, and Athanasios V. Vasilakos, "Routing Metrics of Cognitive Radio Networks: A Survey", IEEE Communications surveys \& tutorials, Vol.16, Issue.1, pp.92-109, 2014.

[5] Amjad Ali, Muddesar Iqbal, Adeel Baig, Xingheng Wang, "Routing Techniques in Cognitive Radio Networks: A Survey", International Journal of Wireless \& Mobile Networks (IJWMN), Vol.3, No.3, June 2011.

[6] Shelly Salim and Sangman Moh, "On-demand routing protocols for cognitive radio ad hoc networks", EURASIP Journal on Wireless Communication and Networking, Article ID: 10499, 2013. doi:doi.org/10.1186/1687-1499-2013-102.

[7] R.Rai, V.Tiwari and S.Kansal, "Comparison of routing protocol AODV and WCETT in Cognitive Radio Networks", International Journal of Engineering
Research \& Technology (IJERT), Vol2, no.10, 2013.

[8] Sajjad Rahmani, M. Afshin Hemmatyar "Performance Evaluation of Transport Layer in Cognitive Radio Adhoc Networks", International Journal of Computer Applications (0975 - 8887) Volume 103 - No.5, October 2014.

[9] Yuting Wang, Guoqiang Zheng, Huahong Ma, Yang Li, and Jishun Li, "A Joint Channel Selection and Routing Protocol for Cognitive Radio Network", Wireless Communication and Mobile Computing, Volume 2018, Article ID 684864, 7 pages. 2018 doi:doi.org/10.1155/2018/6848641

[10] M. C. Oto and O. B. Akan, "Energy-Efficient Packet Size Optimization for Cognitive Radio Sensor Networks," in IEEE Transactions on M. C. Oto and O. B. Akan, "Energy-Efficient Packet Size Optimization for Cognitive Radio Sensor Networks," in IEEE Transactions on Wireless Communications, vol. 11, no. 4, pp. 1544-1553, April 2012.

[11] Mohammed Al-Medhwahi, Fazirulhisyam Hashim, Borhanuddin Mohd Ali and A. Sali "Impact of Packet Size in Adaptive Cognitive Radio Sensor Network" Wireless Communications and Mobile Computing Volume 2018, Article ID 3051204, 9 pages doi:doi.org/10.1155/2018/3051204.

[12] M. Al-Medhwahi, F. Hashim, B. M. Ali, and A. Sali, "Pliable cognitive MAC for heterogeneous adaptive cognitive radio sensor networks," PLoS ONE, vol. 11, no. 6, p. e0156880, 2016.

[13] Richard S. Sutton and Andrew G. Barto. 2018 Reinforcement Learning: An Introduction. A Bradford Book, Cambridge, MA, USA.

[14] S. Talekar and S. Terdal, "Reinforcement Learning Based Channel Selection for Design of Routing Protocol in Cognitive Radio Network", $20194^{\text {th }}$ International Conference on Computational Systems and Information Technology for Sustainable Solutions (CSITSS), Bengaluru, India, 2019, pp. 1-6, doi:10.1109/CSITSS47250.2019.9031024.

[15] "Cognitive Radio Cognitive Network Simulator". [Online]. Available: http://stuweb.ee.mtu.edu/ Ijialian/ 